\title{
Addressing the Challenges of Government Service Provision with Artificial Intelligence
}

\author{
Yongqing Zheng, Han Yu, Lizhen Cui, \\ Chunyan Miao, Cyril Leung, Yang Liu, Qiang Yang
}

\begin{abstract}
In complete contract theory, the main approach to limit moral hazard is through modifying incentives for the agents. However, such modifications are not always feasible. One prominent example is Chinese government service provision. Over the years, it has been plagued with inefficiencies as a result of moral hazard. Previous attempts to address these challenges are not effective, as reforms on civil servant incentives face stiff hindrance. In this article, we report an alternative platform - SmartHS - to address these challenges in China without modifying incentives. Through dynamic teamwork, automation of key steps involved in service provision, and improved transparency with the help of artificial intelligence, it places civil servants into an environment that promotes efficiency and reduces the opportunities for moral hazard. Deployment tests in the field of social insurance service provision in three Chinese cities involving close to 3 million social insurance service cases per year demonstrated that the proposed approach significantly reduces moral hazard symptoms. The findings are useful for informing current policy discussions on government reform in China and have the potential to address long-standing problems in government service provision to benefit almost onefifth of the world's population.
\end{abstract}

\begin{abstract}
7 he organizational structure of the Chinese government follows a top-down, tree-like hierarchy (Horling and Lesser 2005) that is designed to provide effective control. A similar structure can be found within each level of government organization. Under such a structure (figure 1), official communications between leaf nodes need to be routed through their parent nodes, which sacrifices efficiency for better control. Government services (for example, claiming social insurance benefits, setting up an enterprise, and changing residence from one city to another) are superimposed on top of this hierarchical bureaucratic structure. Most of the time, citizens only directly deal with the leaf nodes, which are the executive branches providing government services. One task may require several leaf nodes (not necessarily all from the same organization) to be combined into a workflow (figure 1).
\end{abstract}




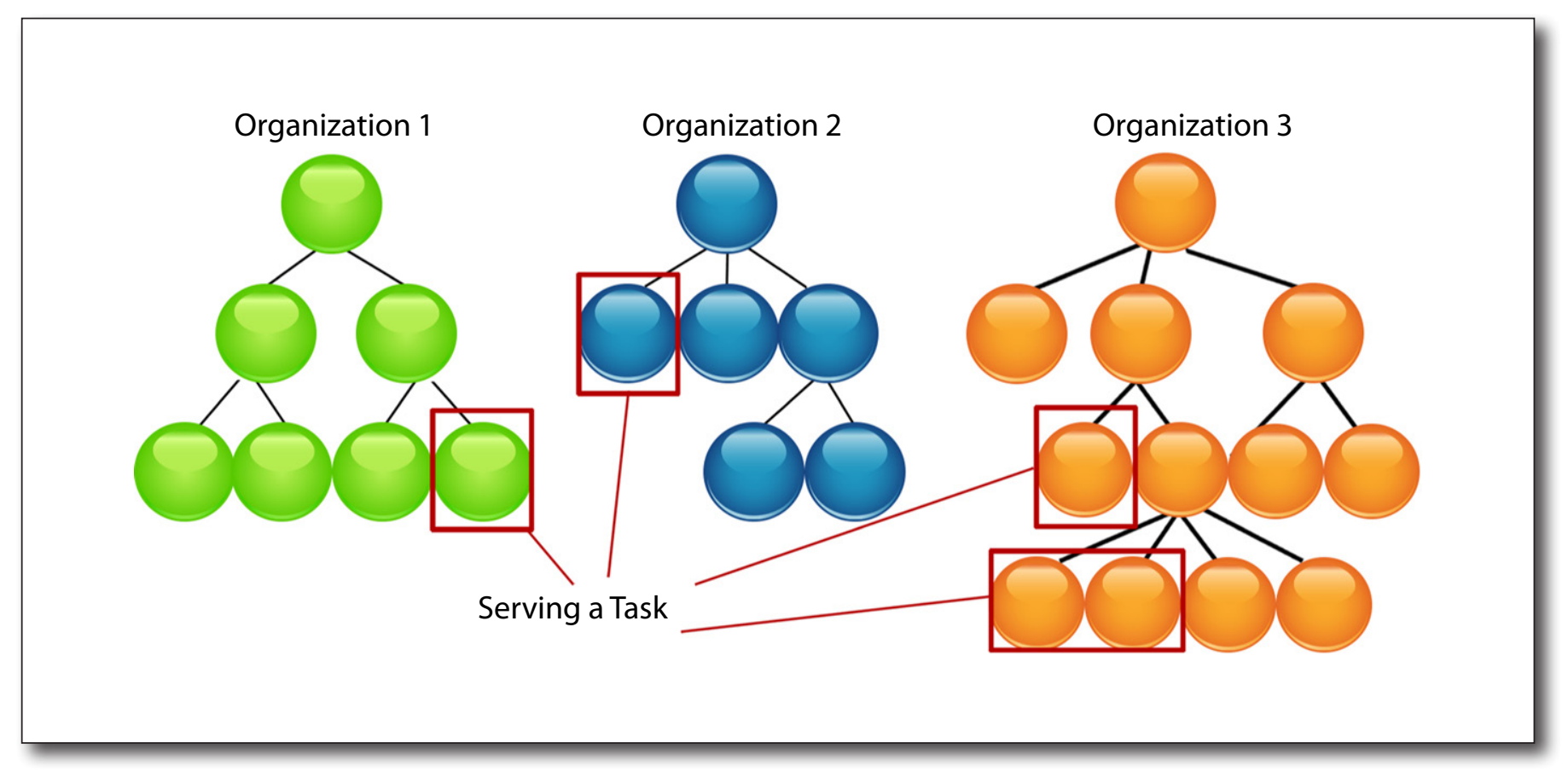

Figure 1. An Example Hierarchical Organizational Structure.

The inefficiency and asymmetry of information in government service provision has given rise to an environment in which moral hazard (Hölmstrom 1979) and even corruption (Jia 2007) frequently occur. It has resulted in undesirable behavior patterns among many civil servants. Today, the problem of providing quality government services is well recognized by the State Council of China. It has issued official reports detailing the difficulties many citizens have experienced and calls for effective solutions (The People's Republic of China 2016). The most frequently mentioned symptoms of moral hazard include procrastination, avoiding responsibility, rent-seeking, abuse of discretion, guanxi (Park and Luo 2001)-based exchange of favors, citizens having to go through multiple government departments for one matter (sometimes repeatedly), and long queues.

The Chinese government has been aware of this situation and recognizes the importance of adopting a service-oriented approach to government. In recent years, efforts to improve accountability from a policy standpoint have permeated into many aspects of public service in China (Nature 2005). Electronic government (e-government) has been identified by China as an important approach to enhance citizens' access to government as well as the government's access to citizens. The application of e-government in countries such as the United States and Singapore has demonstrated great effectiveness in acting as a medium for delivering better services to citizens and improving internal efficiency (Bertot et al. 2010). The currently widely used e-government system in China (Seifert and Chung 2009) did not focus on breaking down the organizational boundaries across multiple government departments which are impeding a task-oriented workflow, but are instead similar to electronic toolboxes focused on improving individual civil servant efficiency (Zhang 2002; Holliday and Yep 2005; Li 2005; Seifert and Chung 2008). Thus, they have been largely unsuccessful in improving poor quality of government services as a result of systemic inefficiency and moral hazard.

To address the limitations of existing Chinese e-government initiatives and improve the quality of government services, we proposed an artificial intelligence (AI)-empowered smart human resource service platform - SmartHS - designed on the complete contract theory (Grossman and Hart 1983) for social insurance government service provision. Through the process of standardizing service provision procedures for many tasks involved in social insurance, we identified two main types of complete contract relationships among the stakeholders. The moral hazard that exists in these relationships is the root cause of many aforementioned characteristics of poor government service provision. The proposed platform is an information and communications platform with workflow powered by AI. It replaces the current rigidorganization-oriented government service provision environment with one that is task-oriented. A workflow is modeled as a series of alternating task execution stages and transition stages.

We incorporated the essence of the complete contract theory into these two stages according to the following approach to improve efficiency and reduce the likelihood for moral hazard:

For the task execution stage, the SmartHS platform provides real-time status information about the 


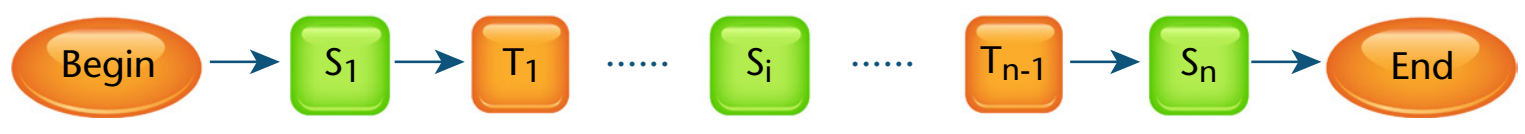

Figure 2. The General Workflow of a Typical e-Government Service in China.

workflow to the task requesters and the government organization in charge, thereby enhancing the observability of the agents' actions and reducing the risk of moral hazard. The SmartHS platform also enables fine-grained performance information tracking and information sharing across organizations to improve information transparency and support data-driven abnormality alerts, interventions, and performance assessments by the agents' superiors, thereby enhancing the fairness and effectiveness of incentive mechanisms and reducing opportunities for moral hazard.

For the transition stage, the SmartHS platform performs the role of the agent to automatically transfer required digital resources to the next task execution stage following predefined system logics, thereby eliminating the possibility of moral hazard.

The proposed platform, however, does not modify the actual incentive mechanisms (e.g., performance evaluation, performance bonus calculation, and promotion) that are currently in place.

Through a large-scale deployment testing of SmartHS in three Chinese cities involving 2,920,000 cases, we found that the key symptom of moral hazard in the public service provision in China - lack of efficiency - has been significantly mitigated, with more than 70 percent of all users choosing to access government services through SmartHS. Although moral hazard cannot be directly measured in practice, the result is a strong indicator that the proposed approach has the potential to significantly improve public service provision. The findings are useful for informing policy discussions on government reform in China.

\section{Method}

In this section, we analyze the challenges facing existing Chinese e-government service provision from the perspective of Contract Theory, and explain how the proposed approach addresses these challenges by fundamentally changing the environmental conditions under which civil servants work and interact across organizational boundaries.

\section{Existing e-Government Service Provision Systems in China}

In general, a government service workflow, which consists of $x$ intermediate steps, is illustrated in figure
2. $S_{i}$ denotes the $i$ th step (that is, an execution stage) involved in the workflow, whereas $T_{i}$ (a transition stage) denotes the transition between $S_{i}$ and $S_{(i+1)}$. $T_{i}$ often involves passing the output of $S_{i}$ (for example, data, documents, and proof of authorization) to $S_{(i+1)}$. Because we are dealing with government services that have precisely defined prerequisites, rules, and regulations for each $S_{i}$, the relationship between the civil servant responsible for each $S_{i}$ (that is, the agent) and his or her superior (the principal) can be modeled as a Complete Contract relationship (Grossman and Hart 1983). Similarly, the relationship between the agent for $S_{i}$ and the agent for $S_{(i+1)}$ can also be modeled as a Complete Contract relationship. Thus, the workflow of a government service can be perceived as a chain of contracts.

Table 1 summarizes the elements involved in the two contract relationships we have identified during the execution stage and the transition stage. It can be observed that the definition of the contract terms and the principal-agent relationships are clear. However, the main problem lies in the observability of actions and the lack of authority to award incentives or to intervene. These conditions have given rise to severe moral hazard in current Chinese government service provision. To illustrate where moral hazard can occur, we use an example scenario of a resident filing a social insurance benefits claim using the traditional e-government system in China (as shown in figure 3a).

In this scenario, the resident has to go to the front desk of a service center as his or her identity and employment relationship documents need to be verified first. This information has not been integrated into the traditional Chinese e-government social insurance claim system, and it has to be repeatedly verified for each case. Once this step is completed, the supporting documents, together with an application form, are passed on to the data entry clerk who uses a traditional e-government tool to digitize these documents. Nevertheless, this step is for the purpose of making archiving easier, and the original documents still need to be delivered physically to the next step in the workflow, which is the calculation of benefits. In this instance, the data entry clerk is put in a position of power to determine how many cases shall accumulate at the data acquisition step before he or she should make a batch delivery to the 
Execution Stage

Principal

Agent

Contract

Incentives

Observability

Basis to Determine Incentives

Authority to Award Incentives

Intervention

Potential for Moral Hazard
The superior of the civil servant in charge

The civil servant in charge

The definitions of executing a step

Bonus and promotion mechanisms

Only through random sampling

Very limited

Yes

To some extent

High
Transition Stage

The civil servant in charge of the next execution stage

The civil servant in charge of the previous execution stage

The definitions of how information shall pass between steps

Bonus and promotion mechanisms

None

Very limited

No

No authority

Very high

Table 1. Complete Contract Relationships in Traditional Chinese e-Government Service Provision.

benefits calculation office. He or she can also determine, to some extent, which incoming case to enter into the system first. Such decisions can theoretically be made based on considerations for efficiency, but in practice, are often influenced by discretion and possibly moral hazard. This similar situation repeatedly occurs in each step down the line of this workflow. The civil servants in charge of benefits calculation, approval, and payment are all put into implicit positions of power as a result of the inefficiency in the system itself. Thus, they are exposed to many opportunities of discretion. The only one who is exposed to fewer opportunities of discretion is the one in charge of archiving as the transaction with the resident effectively ended before this step, and there is no subsequent step depending on it.

\section{Changing the Environment of Gov- ernment Service Provision with SmartHS}

To address these challenges, the key is to change the environment in which civil servants work to minimize the inefficiencies that give rise to opportunities for moral hazard. From a technical perspective, SmartHS relies on the state of the art but does not advance it. Technologies such as cloud computing (Armbrust et al. 2010), blockchain for distributed data security (Swan 2015), big data analytics (Zikopoulos and Eaton 2011) and AI optimization techniques (Marler and Arora 2004) have been used to realize system functionalities. It is how SmartHS redesigned the complete contract relationships in government service provision with the help of AI scheduling that mitigated moral hazard.

Table 2 summarizes the elements involved in the revised complete contract relationships under SmartHS. The items that differ from the corresponding items in table 1 are highlighted in bold. The key changes are twofold: automating all the transition stages, and improving observability. These changes, which can be implemented easily with today's technologies, have profound implications on the characteristics of government service provision.

Figure $3 \mathrm{~b}$ illustrates how the same scenario under figure 3a plays out under SmartHS. This time, the resident has more choices for accessing the services. If his or her personal information has already been verified and saved into the system (possibly through prior interactions), he or she can use networked devices via personal Internet (PI) or workplace Internet (WI), or the Self-service Kiosks (SKs) placed at the service centers (SCs) to file his or her claims. Otherwise, he or she can still submit his or her claim through traditional means in SCs in urban areas or community service centers (CSCs) in rural areas. Once the case is entered into the system, the digitized information is automatically routed to the civil servants in charge based on its current processing status. Information required to perform the task at each step is automatically assembled by the system based on policy parameters, the standardized workflows, and the civil servants' roles. Once a task is marked as completed, it is automatically routed to the next step with workload optimization to wait for further processing.

The analytical framework that we developed to derive the solution is illustrated in figure 4 . In this framework, individual civil servants' (that is, the trustees') key performance indicators including their reputation values and capacity limits are tracked by the system based on their past actions. Situational factors including the value of the tasks and each civil servant's current workload are also available in the SmartHS platform. The framework enables individual well-being and system-level well-being to be jointly modeled into one objective function, which can be solved efficiently with a polynomial time solution 


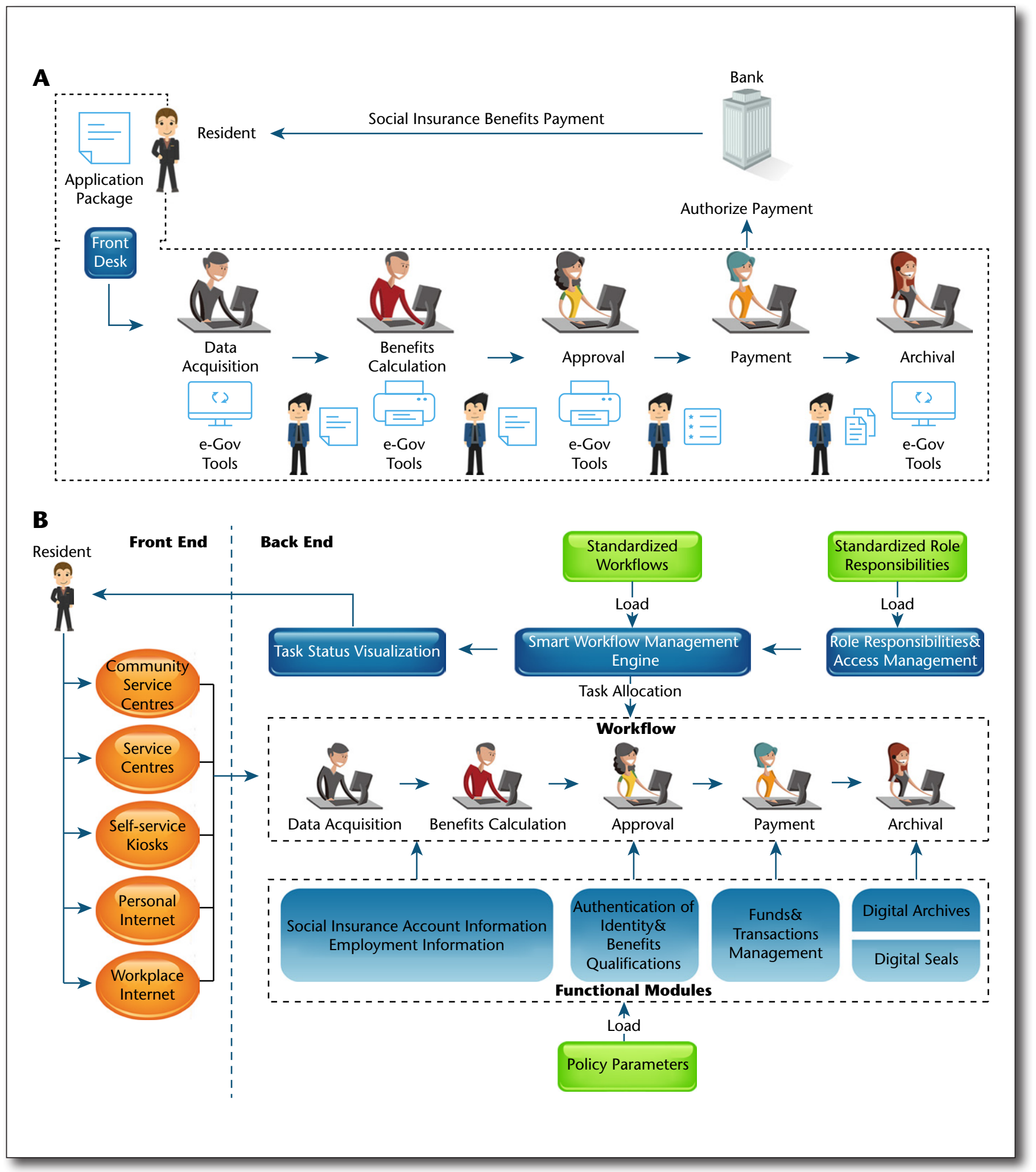

Figure 3. An Example Scenario of a Resident Filing a Social Insurance Benefits Claim.

(a) The process under traditional Chinese e-government systems. (b) The process under the proposed SmartHS platform. 
Execution Stage

The superior of the civil servant in charge

The civil servant in charge

Agent

The definitions of executing a step

Contract

Incentives

Observability

Basis to Determine Incentives

Authority to Award Incentives

Intervention

Potential for Moral Hazard
Bonus and promotion mechanisms

Fine-grained performance tracking

Data-driven

Yes

Data-driven

Relatively low
Transition Stage

The civil servant in charge of the next execution stage

SmartHS

The definitions of how information shall pass between steps

Not necessary

Fine-grained performance tracking

Not necessary

No

No authority

None

Table 2. Complete Contract Relationships in SmartHS.

approach based on indexing. The AI-powered task delegation optimization approach is presented in detail in Yu et al. (2017), which is ethically aligned (Yu et al. 2017; Yu, Shen, Miao, Leung, Lesser, Yang 2018) to dynamically adjust the priority of workers' well-being and system efficiency in a situation-aware manner. It plays a key role in enabling SmartHS to dynamically adapt to complex changes in civil servants' availability as well as variations in workload to maintain a high task throughput in the system. Through automated task delegation, the system also eliminates an important source of potential moral hazard. More details on the AI task delegation engine in SmartHS can be found in Zheng et al. (2018).

By providing a shared information infrastructure about users' identity, employment, and other important information related to social insurance claims in a secure and authenticated way, the transition stage can be fully automated. This circumvents the need for incentives to improve efficiency in this stage, and eliminates the potential moral hazard facing civil servants who used to be forced to determine how frequently to deliver required documents to the next step in a given workflow. As SmartHS tracks, in detail, the amount of time spent on serving each case at each step, it offers fine-grained observability to the principals (including the civil servants' superiors and the overall principal - the citizens requesting the services). This offers the superiors a data-driven mechanism, which helps them determine the distribution of incentives objectively and fairly. The time taken to serve a case can also be compared with the average time taken by SmartHS automatically to identify abnormalities and initiate interventions. SmartHS changes the organization of civil servants from implicit positions of power to roles, and reduces the opportunities for moral hazard to occur (in the case of the transition stages, completely eliminates moral hazard).

\section{Implications}

In this section, we analyze the effects of SmartHS on the quality of social insurance services delivered to the residents one year after it has been deployed in three Chinese cities - Dongying, Weihai, and Yantai. Where possible, we will compare the performance achieved by SmartHS with data from these three cities before its deployment. Data from seven other cities (Binzhou, Dezhou, Jining, Laiwu, Taian, Weifang, and Yingkou) with comparable population sizes and levels of development with these three cities are also used in the comparison.

Figure 5a shows the average time taken for the 10 most frequently used services with and without SmartHS. For the case under SmartHS, the average time is computed with data from the three cities where it has been deployed. For the case without SmartHS, the average time is computed with data from all 10 selected cities. The 10 services are ranked in descending order of the average time taken without SmartHS. It can be observed that under SmartHS, the average time taken to serve these frequently used services has decreased significantly. Without using SmartHS, each of these 10 services requires 35 days on average to be completed. With SmartHS, this average time is reduced to 14 days, reducing waiting time by 59 percent.

Figure $5 \mathrm{~b}$ illustrates the cumulative percentage of services completed over different lengths of time for all social insurance services. It can be observed that within 2 weeks' time, the traditional e-government system in China is able to process 22 percent of the requests, whereas SmartHS is able to process 38 percent of the requests. Under SmartHS, 67 percent of all services can be completed within one month from the date of being requested, whereas the traditional e-government system in China can only 


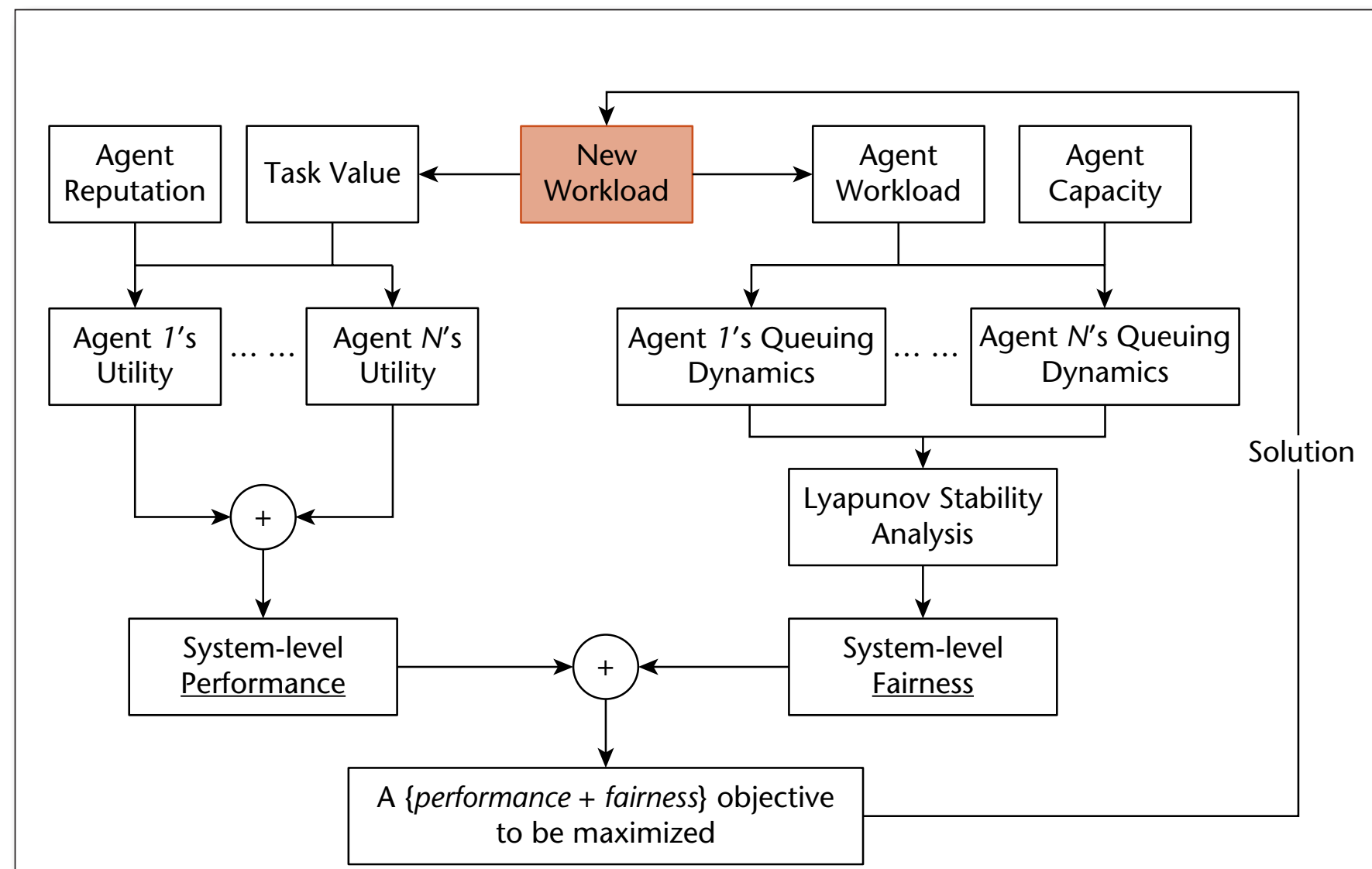

Figure 4. The Analytical Framework Behind the Task Allocation Optimizer in the SmartHS AI Engine.

complete 31 percent during the same length of time. All service requests under SmartHS can be completed within 100 days. However, all service requests can only be completed within 210 days under the traditional e-government system in China.

Figure $5 \mathrm{c}$ shows the breakdown of the average time taken for the different stages involved in the workflows for service provision under SmartHS. Tasks in SmartHS can be divided into two categories based on how they are processed: batch tasks, which require someone in a position of authority to process multiple tasks in one pass (for example, payment authorization); and individual tasks, which can be served immediately by more than one qualified civil servant that the system can dynamically match the task to, who is immediately available to process. Once a task is assigned to an agent by SmartHS, it stays pending in his or her backlog until he or she starts to process it. This stage is referred to as the Pending stage in figure $4 \mathrm{c}$. For batch tasks, the average pending time is around 2 days and the average processing time is 6.5 days. For individual tasks, the average pending time is around 2 seconds and the average processing time is 93 seconds. Once the tasks are completed, they are automatically forwarded to the next step in the workflow by SmartHS. Thus, the average transition time depends only on the system processing time and the network transition time (between 3 and 5 seconds for both batch tasks and individual tasks).

The analysis of the advantages of SmartHS focuses on improvement in efficiency and reduction in time elapsed. The reasons are twofold. Firstly, time spent on various stages of service provision is the main metric being tracked by SmartHS. It is also the main metric used to determine if any abnormality has occurred and whether interventions are required. Secondly, changes in service provision time can indirectly reflect how conducive the operating environment is to moral hazard. The phenomenon of moral hazard is very difficult to study directly, as civil servants are strongly motivated not to admit having engaged in such behaviors. Therefore, changes in work efficiency measured by time elapsed is selected in this research as a surrogate to study this phenomenon indirectly. By eliminating the need for manually passing documents and information from stage to stage, civil servants do not need to determine when to pass which cases along and are, therefore, free from moral hazard during transitions. By organizing civil servants dynamically based on different workflows, they 
A

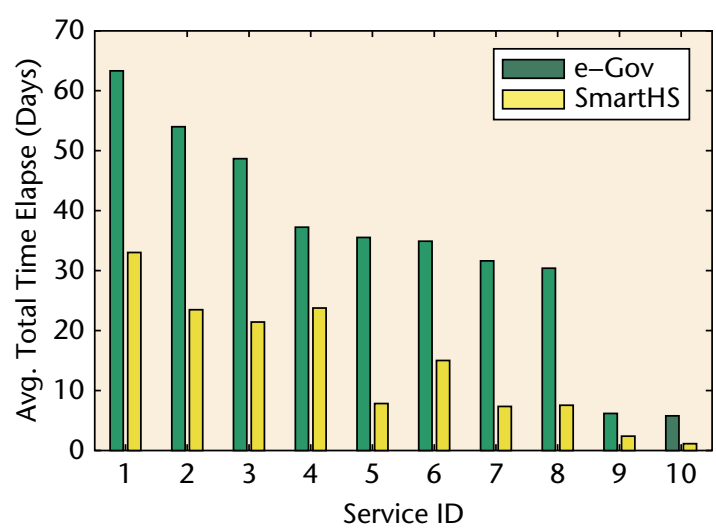

C

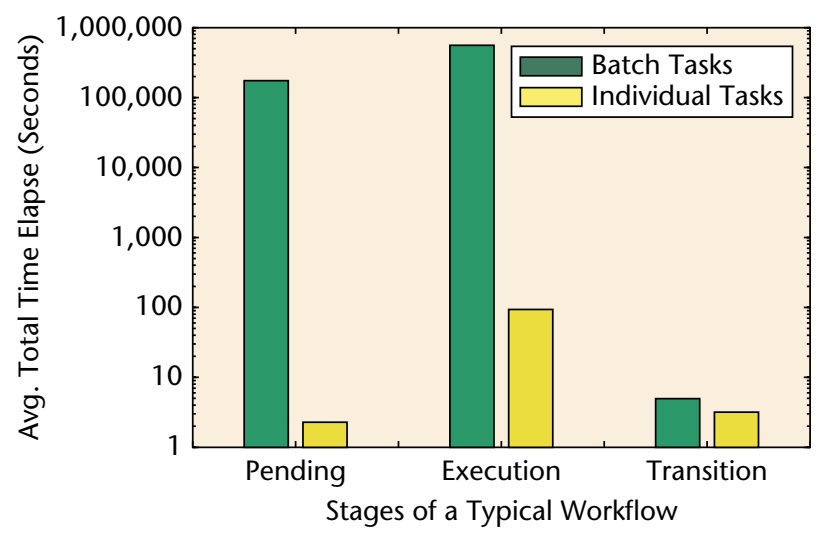

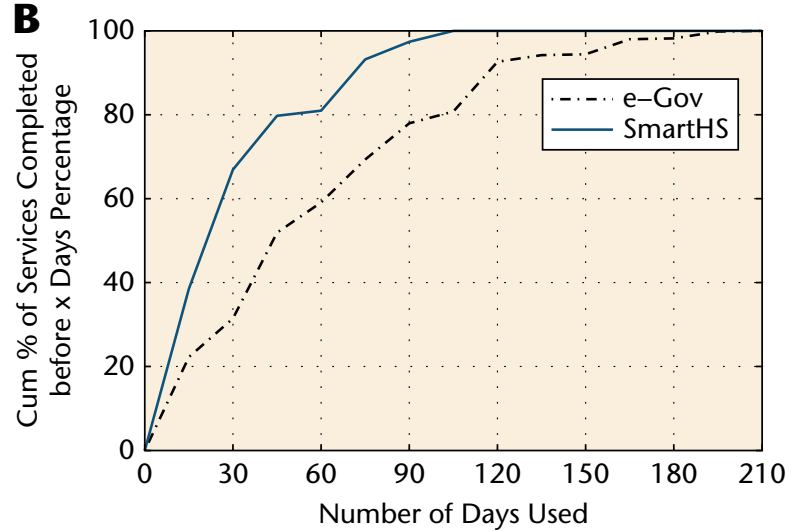

D

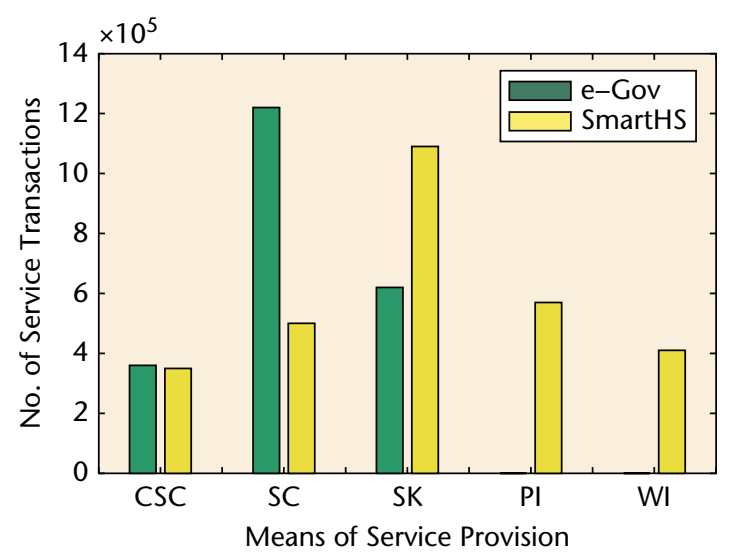

Figure 5. Results.

(a) The average time taken for 10 most frequently used social insurance benefits claim services in 10 Chinese cities, including Binzhou, Dezhou, Dongying, Jining, Laiwu, Taian, Weifang, Weihai, Yantai, and Yingkou. (b) The cumulative percentage of services completed within $x$ days. (c) The average time spent on different stages of a typical Chinese social insurance service workflow expressed in seconds (note: the $y$-axis is drawn in logarithmic scale). (d) The usage patterns for different means of accessing the Chinese social insurance services (CSCs, SCs, SKs, PI, and WI).

resemble more closely workers on an assembly line. The reduction of average processing time of individual tasks down to less than 2 minutes under SmartHS reflects, to some extent, fewer opportunities for moral hazard.

Figure $5 \mathrm{~d}$ illustrates the different usage patterns under SmartHS and the traditional social insurance e-government system in China. The data for this figure are only from the three cities currently using SmartHS so as to reflect changes in the usage patterns before and after the deployment of SmartHS. Before SmartHS, using the traditional e-government system, residents had been accessing social insurance government services manually through SCs (located in municipal areas), CSCs (located in rural areas), or SKs (standalone systems). With the arrival of SmartHS, even the SKs were upgraded to the SmartHS system.
As SmartHS can be accessed via any Internet-enabled device, residents can also use their PI or WI to obtain social insurance government services. During the one-year period before the deployment of SmartHS, CSCs served 360,000 requests. CSCs served the greatest number of requests numbering 1,220,000. Another 620,000 requests were served by SKs.

During the one-year period after the deployment of SmartHS, CSCs served 350,000 requests, which is similar to the amount of service before. This can be attributed to the relatively low level of Internet penetration in rural areas in China where the residents have to access government services through service front desks. There was a significant drop in the number of services accessed through SCs from 1,220,000 to 500,000 after the deployment of SmartHS. SKs now attract the greatest number of uses at 1,090,000. 
The number of services accessed via PI with SmartHS is 570,000 , whereas that via WI is 410,000 . Before SmartHS, only 28 percent of all the requests were served through kiosks equipped with the traditional e-government system. With SmartHS, 71 percent of all the requests are served via networked interactions (SKs, PI, and WI). Under SmartHS, an additional 720,000 services have been provided to residents a 33 percent increase over those received from the traditional e-government system.

\section{Discussion}

Long waiting time, poor quality of service, lack of transparency on progress, and widespread moral hazard have been major factors tarnishing the reputation of Chinese government service provision. Although it has been recognized by many researchers that the solution potentially lies in applying Contract Theory into such situations, there is a lack of effective and practical solutions that can be deployed in China. Changing the expected payoff for the civil servants (i.e., agents) involved in these situations often require reforming the incentive mechanisms and other sensitive measures that face strong resistance in Chinese government bureaucracy today.

In this article, we report our experience addressing these challenges using a technology-centric approach. By identifying key stages in government service provision, we developed an AI-powered e-government service platform - SmartHS - to infuse complete contract relationships into government service provision. Without modifications to the existing incentive structures in Chinese government organizations, the system is perceived to be more acceptable by the civil servants and has so far been deployed in three cities in China. However, the platform changes the environment in which civil servants work in three ways: Dynamic collaboration, automating the transition state, and improved transparency.

The first way is through dynamic collaboration. The platform takes a workflow-oriented approach of service provision in which a civil servant is dynamically assigned different roles under different workflows. This breaks down organizational boundaries and streamlines collaboration among civil servants in charge of different duties across multiple government departments for improved efficiency.

The second way is by automating the transition stage. By digitizing required documents for, and the results produced by, each execution stage, SmartHS is able to remove the human elements required by the traditional Chinese e-government system to pass documents and information along a workflow. This removes an important link in which discretion can potentially be abused, and eliminates the opportunity for moral hazard to occur during the transition stages.

The third way is through improved transparency. The SmartHS platform monitors the performance of each civil servant at each stage in detail by measuring the time taken to process the tasks and comparing it against various average metrics. This provides the civil servants' superiors with data-driven objective measures to determine how incentives shall be disbursed, and enables the system to generate alerts if abnormalities have been detected. By making the civil servants aware that such monitoring is being conducted, they are motivated to provide better quality service and reduce moral hazard behaviors.

The observed data from actual operation demonstrate significantly improved efficiency. From another angle, the improved efficiency means that civil servants spend less time than before on each of the task assigned to them, giving them fewer opportunities to engage in moral hazard behaviors. The proposed platform and approach represent a successful attempt to circumvent sensitive restrictions on government service provision reforms toward building a serviceoriented government in China. Within the new operating environment provided to the civil servants by SmartHS, the citizens, the civil servants, and the overseeing authorities are organized into more symbiotic relationships. This forms the foundation for sophisticated incentive mechanisms (Weyl et al. 2010) and work-life-balance scheduling techniques (Yu et al. 2019) to be explored in the context of government service provision.

\section{Moving Forward}

With increasing public awareness of the need for AI-based systems to respect and protect user privacy, strict privacy protection legislations are emerging in the European Union (European Union, Parliament and Council 2016), the US, and China. As government service provision typically involves multiple agencies with each storing a different set of data concerning certain aspects of a particular citizen, the need for privacy protection and compliance with data privacy laws is imperative.

In response to the new privacy-conscious legal environment, the AI research community has proposed a new paradigm of machine learning - federated machine learning (FML) - in which a group of data owners collectively train a model while keeping their data stored locally. Secure protocols such as homogeneous encryption, differential privacy, and secret sharing are used to ensure that the information shared among federation participants (e.g., model loss and gradients) does not breach user privacy (Yang et al. 2019).

We plan to collaborate with the Federated AI Ecosystem, which is spearheading a global open initiative to carry out research, development, and deployment of FML, to strengthen the privacy protection aspect of SmartHS. By making available source code for the building blocks of FML through Federated AI Technology Enabler and working on establishing a FML Standard with the IEEE (IEEE P3652.1), Federated AI Ecosystem is providing a focal point for the AI research community to sustain the strong development trajectory of AI moving forward even as the 
legal environment becomes tougher. We foresee that such a collaboration to further improve SmartHS with FML will bring increased benefit for citizens accessing government services online in China.

\section{Acknowledgments}

This research is supported by the Shandong Province Independent Innovation Project: the Research, Development and Promotion of Smart Social Security Platform (No. 2013CXC30201); the National Research Foundation, Prime Minister's Office, Singapore under its Interactive Digital Media Futures Funding Initiative; and Nanyang Technological University's Nanyang Assistant Professorship.

\section{References}

Armbrust, M.; Fox, A.; Griffith, R.; Joseph, A. D.; Katz, R.; Konwinski, A.; Lee, G.; Patterson, D.; Rabkin, A.; Stoica, I.; and Zaharia, M. A. 2010. View of Cloud Computing. Communications of the ACM 53(4): 50-8. doi.org/10.1145/ 1721654.1721672.

Bertot, J. C.; Jaeger, P. T.; and Grimes, J. M. 2010. Using ICTs to Create a Culture of Transparency: E-Government and Social Media as Openness and Anti-Corruption Tools for Societies. Government Information Quarterly 27(3): 26471. doi.org/10.1016/j.giq.2010.03.001.

European Union, Parliament and Council. 2016. Regulation (EU) 2016/679 of the European Parliament and of the Council of April 27, 2016 on the Protection of Natural Persons with Regard to the Processing of Personal Data and on the Free Movement of Such Data, and Repealing Directive 95/46/ EC. Official Journal of the European Union L 119/1 April 27. https://eur-lex.europa.eu/legal-content/EN/TXT/?uri= celex\%3A32016R0679.

Grossman, S. J., and Hart, O. D. 1983. An Analysis of the Principal-Agent Problem. Econometrica 51(1): 7-46. https:// EconPapers.repec.org/RePEc:ecm:emetrp:v:51:y:1983:i:1: p:7-45.

Holliday, I., and Yep, R. 2005. E-government in China. Public Administration and Development 25(3): 239-49. doi. org/10.1002/pad.361.

Hölmstrom, B. 1979. Moral Hazard and Observability. The Bell Journal of Economics 10(1): 74-91. doi.org/10.2307/3003320.

Horling, B., and Lesser, V. R. 2005. A Survey of Multi-Agent Organizational Paradigms. The Knowledge Engineering Review 19(4): 281-316. doi.org/10.1017/S0269888905000317.

Jia, H. 2007. China Syndrome - a Regulatory Framework in Meltdown? Nature Biotechnology 25(8): 835-7. doi.org/ 10.1038/nbt0807-835.

Li, B. 2005. On the Barriers to the Development of EGovernment in China. In Proceedings of the 7th International Conference on Electronic Commerce, 549-552. New York: Association for Computing Machinery. doi.org/10.1145/ 1089551.1089650.

Marler, R., and Arora, J. 2004. Survey of Multi-Objective Optimization Methods for Engineering. Structural and Multidisciplinary Optimization 26(6): 369-95. doi.org/10.1007/ s00158-003-0368-6.

Nature. 2005. Editorial, New Accountability in China. Nature 434(7037): 1053. doi.org/10.1038/4341053b.

Park, S. H., and Luo, Y. 2001. Guanxi and Organizational Dynamics: Organizational Networking in Chinese Firms.
Strategic Management Journal 22(5): 455-77. doi.org/10.1002/ smj.167.

Seifert, J. W., and Chung, J. 2009. Using E-Government to Reinforce Government-Citizen Relationships: Comparing Government Reform in the United States and China. Social Science Computer Review 27(1): 3-23. doi.org/ 10.1177/0894439308316404.

Swan, M. 2015. Blockchain: Blueprint for a New Economy. Sebastopol, CA: O'Reilly Media.

The People's Republic of China. 2016. The Guidance of the State Council on Accelerating the Work of Internet Government Services. Technical Report. Beijing, China: The State Council of the People's Republic of China.

Weyl, E. G.; Frederickson, M. E.; Yuc, D. W.; and Pierce, N. E. 2010. Economic Contract Theory Tests Models of Mutualism. Proceedings of the National Academy of Sciences of the United States of America 107(36): 15712-6. doi.org/10.1073/ pnas.1005294107.

Yang, Q.; Liu, Y.; Chen, T.; and Tong, Y. 2019. Federated Machine Learning: Concept and Applications. ACM Transactions on Intelligent Systems and Technology 10(2): Art. 12. doi.org/10.1145/3298981.

Yu, H.; Miao, C.; Chen, Y.; Fauvel, S.; Li, X.; and Lesser, V. R. 2017. Algorithmic Management for Improving Collective Productivity in Crowdsourcing. Scientific Reports 7(1): 12541. doi.org/10.1038/s41598-017-12757-X.

Yu, H.; Miao, C.; Zheng, Y.; Cui, L.; Fauvel, S.; and Leung, C. 2019. Ethically Aligned Opportunistic Scheduling for Productive Laziness. In Proceedings of the 2nd Association for the Advancement of Artificial Intelligence/Association for Computing Machinery Conference on Artificial Intelligence, Ethics, and Society (AIES-19). New York: Association for Computing Machinery. doi.org/10.1145/3306618.3314240.

Yu, H.; Shen, Z.; Miao, C.; Leung, C.; Lesser, V. R.; and Yang, Q. 2018. Building Ethics Into Artificial Intelligence. In Proceedings of the 27th International Joint Conference on Artificial Intelligence (IJCAI'18), 5527-5533. https://arxiv. org/abs/1812.02953.

Zhang, J. H. 2002. Will the Government Serve the People? The Development of Chinese E-Government. New Media \& Society 4(2): 163-84. doi.org.10.1177/14614440222226325. Zheng, Y.; Yu, H.; Cui, L.; Miao, C.; Leung, C.; and Yang, Q. 2018. SmartHS: An AI Platform for Improving Government Service Provision. In Proceedings of the 30th Association for the Advancement of Artificial Intelligence Conference on Innovative Applications of Artificial Intelligence (IAAI-18), 7704-11. Palo Alto, CA: AAAI Press.

Zikopoulos, P., and Eaton, C. 2011. Understanding Big Data: Analytics for Enterprise Class Hadoop and Streaming Data. New York: McGraw-Hill Osborne Media.

Yongqing Zheng is the chief executive officer and deputy chairman of Shanda Dareway Software Pte Ltd. He is also a professor at the School of Software Engineering, Shandong University, China. His research focuses on AI and the application of this technology to solve real-world problems.

Han Yu is a Nanyang assistant professor at the School of Computer Science and Engineering, Nanyang Technological University, Singapore. He obtained his PhD from the School of Computer Science and Engineering, Nanyang Technological University. His research focuses on real-time, AI-powered, data-driven algorithmic management for complex collaborative networks. He has published over 100 research papers in the form of book chapters; proceedings 
for leading international conferences such as the Association for the Advancement of Artificial Intelligence, the International Joint Conferences on Artificial Intelligence, and the International Conference on Autonomous Agents and Multiagent Systems; and as articles in journals including AI Magazine, Modern Machinery Science Journal, ACM/ IEEE Transactions, and Scientific Reports. His research work has won 11 conference and journal awards.

Lizhen Cui is a professor and the vice chair of the School of Software Engineering, Shandong University, China. Between 2013 and 2014, he was a visiting scholar at the Georgia Institute of Technology in the United States. His main research interest includes data science and engineering, intelligent data analysis, service computing, and collaborative computing.

Chunyan Miao is a chair professor at the School of Computer Science and Engineering, Nanyang Technological University, Singapore. She received her BSc degree from Shandong University, and her MEng and PhD from Nanyang Technological University. She is the Founding Director of the LILY Research Centre and the Alibaba-Nanyang Technological University Joint Research Institute. Her research focuses on humanized AI.

Cyril Leung is a professor at the Department of Electrical and Computer Engineering at the University of British Columbia, Vancouver, Canada. He received his BSc (first class honors) degree from Imperial College, University of London, England, and his MS and PhD degrees in electrical engineering from Stanford University. His research interests include digital communications, wireless communication networks, sensor networks, ubiquitous computing, elderly-friendly technologies, digital/social signal processing, security and privacy, trust computational models, and information theory.

Yang Liu is a senior researcher in the AI Department of WeBank, China. Her research interests include machine learning, federated learning, transfer learning, multi-agent systems, statistical mechanics, and applications of these technologies in the financial industry. She received her PhD from Princeton University in 2012 and her Bachelor's degree from Tsinghua University in 2007. She holds multiple patents. Her research has been published in leading scientific journals such as ACM Transactions on Intelligent Systems and Technology and Nature.

Qiang Yang is a chair professor at the Department of Computer Science and Engineering of the Hong Kong University of Science and Technology. He received his MSc degree in Astrophysics, MSc in Computer Science, and PhD in Computer Science at the University of Maryland in the US. His research interests include transfer learning, machine learning, planning, and data mining in AI. He is also the president of the International Joint Conferences on Artificial Intelligence, an executive council member of the Association for the Advancement of Artificial Intelligence, and editor-in-chief of IEEE Transactions on Big Data. He was the founding editor-inchief of ACM Transactions on Intelligent Systems and Technology. He was the founding director of the Hong Kong University of Science and Technology's Big Data Institute. He is a fellow of the Association for Computing Machinery, the Institute of Electrical and Electronics Engineers, the Association for the Advancement of Artificial Intelligence, the American Association for the Advancement of Science, the International Association for Pattern Recognition, and the Chinese Association for Artificial Intelligence.

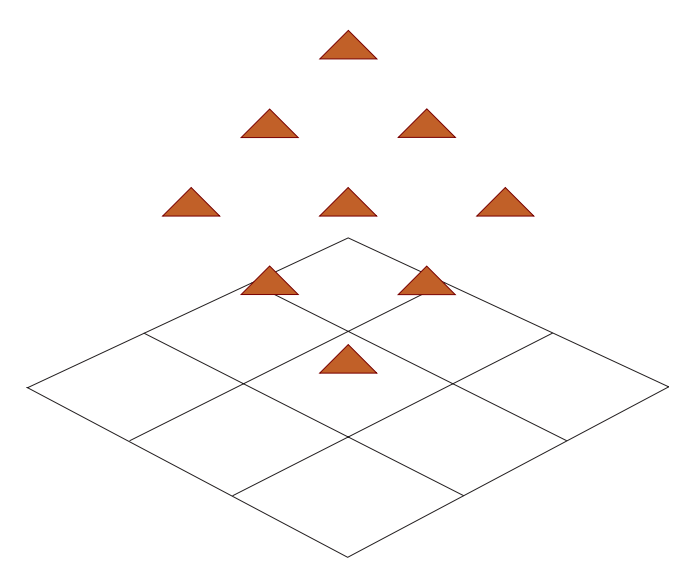

\section{AAAI Fall Symposium Series Call for Proposals}

AAAI is currently accepting proposals for the 2020 Fall Symposium Series, to be held during a combined Spring-Fall Symposium November 11-14 at the Westin Arlington Gateway in Arlington, Virginia adjacent to Washington, DC. The Fall Symposium Series provides the Al community with a unique venue in which researchers from different areas of Al can present speculative work and work in progress, hold focused discussions over several days, build new communities for emerging disciplines, and build ties between existing disciplines.

For complete information on proposal requirements, please see aaai.org/Symposia/Fall/fss20.php. Please submit your symposium proposals to the EasyChair submission site (easychair.org/conferences/ ?conf=fss20) no later than April 30. General inquiries regarding the symposium series should be directed to AAAI:

fss20@aaai.org. 